\title{
Tromboembolismo pulmonar: evolución a largo plazo y epidemiología clínica
}

\author{
M. ECHEGARAY AGARA, J. L. ALONSO MARTÍNEZ, M. A. URBIETA \\ ECHEZARRETA, M. L. ABÍNZANO GUILLÉN, C. GONZÁLEZ ARENCIBIA, \\ M. SOLANO REMÍREZ
}

Servicio de Medicina Interna. Hospital de Navarra. Pamplona. Navarra

PULMONARY THROMBOEMBOLISM: LONG-TERM CLINICAL COURSE AND CLINICAL EPIDEMIOLOGY

\section{RESUMEN}

Antecedentes: La evolución a largo plazo del tromboembolismo pulmonar (TEP) no esta bien establecida.

Material y métodos: Estudio prospectivo observacional (mayo-1992 a diciembre-2002) de pacientes ingresados en un área clínica de M. Interna por TEP con los objetivos de observar la supervivencia, las recidivas, la tasa de hemorragias mayores y la aparición de nuevas neoplasias.

Resultados: Ingresaron 116 pacientes (edad media $72 \pm 11$ años, varones $57-54 \%$-). fallecieron durante el episodio índice 4 pacientes (mortalidad hospitalaria 3,7\%). Diez pacientes fueron perdidos durante el seguimiento. Los 102 pacientes restantes fueron seguidos durante 31.81 \pm 31.23 meses. La tasa de recidiva fue de $19,6 \%$ que ocurrió $22,64 \pm$ 24,57 meses después. La tasa de hemorragia mayor fue de $10,4 \%$. Se diagnosticaron $14(13,7 \%)$ nuevas neoplasias en el seguimiento. La prevalencia total de cáncer asociado a TEP fue $31 \%$.

La mortalidad global fue de $37 \%$, (mayor en mujeres $p<0,01$ ) siendo las principales causas el cáncer (32\%) y el propio TEP más las complicaciones del tratamiento (24\%). La mitad de los fallecimientos ocurrieron en los primeros 12 meses, teniendo una supervivencia acortada para este periodo los pacientes con cáncer $(\mathrm{p}=0,02)$ y los pacientes con recidiva del TEP $(p=0,06)$. La mortalidad posterior al primer año declina al $10 \%$ por año y se hace estable.

Asociados a aumento de la mortalidad estuvieron: edad $>75$ años $(\mathrm{p}<0.001)$, sexo femenino $(\mathrm{p}<0,01)$, pulso $>90 \quad(\mathrm{p}<0,05)$, taquipnea $(\mathrm{p}<0,01)$, retraso en el ingreso y tratamiento hospitalario $(\mathrm{p}<0,05)$, $\mathrm{LDH}>$ a $250 \mathrm{UI}(\mathrm{p}<0,01)$ todos ellos en el episodio índice y la existencia de un cáncer asociado $(\mathrm{p}<0,05)$. En el modelo de regresión logística los factores predictivos de mortalidad fueron la edad, el retraso en el ingreso y los niveles de LDH.

Conclusiones: Aunque la mortalidad por TEP es baja en el episodio índice, a largo plazo es elevada, existiendo un periodo crítico los primeros 12 meses, siendo las principales causas de mortalidad el cáncer y el propio TEP o las complicaciones del tratamiento, disminuyendo y estabilizándose la mortalidad a plazos más largos.

Pueden predecir mortalidad a largo plazo la edad avanzada, el retraso en el diagnóstico y tratamiento y el nivel de LDH del episodio índice.

PALABRAS CLAVE: Tromboembolismo pulmonar. Enfermedad tromboembólica venosa. Curso clínico. Epidemiología.

\section{ABSTRACT}

Background: Long-term clinical course of pulmonary thromboembolism is not well-known. Our aim was to know the events which occur to in-patients diagnosed of pulmonary embolism.

Methods and patients: This is a prospective observational study from May-92 to December-2002 with all in-patients diagnosed of pulmonary thromboembolism at a clinical area of Internal Medicine. Main targets were to know survival, relapses, major hemorrhage rate (Defined as those episodes of bleeding which needed blood transfusion and readmission) and cancer associated rate (Previous and newly diagnosed cancer). Follow up were carried out with telephone contacts with patients and relatives in case of death, and with the computerized system of patients and clinical events of Health Service of Navarra.

Results: One hundred and sixteen patients were included in the study (Mean age 72 SD 11 years male 54\%).During index episode 4 (3.7\%) patients dead. Ten patients were lost in follow up. The rest 102 patients were traced for 31.81 SD 31.23 months (Range 1-127). Relapse rate was 19.6\% that occurred 22.64 SD 24.57 (Range 1-73) months after index episode (Twelve pulmonary embolisms, 5 deep venous thromboses and 3 sudden death with dyspnea). Major hemorrhage rate was $10.4 \%$.

During follow up $14(13.7 \%)$ new cancers were diagnosed (Lung 4 , prostate 2, bladder 2, and colorectal, ovary, breast, liver and kidney one each one). At all prevalence of cancer associated with pulmonary thromboembolism was $31 \%$

Mortality rate was 37\% (Men 25\%, women 49\%, p<0.01). Main causes of death were cancer (32\%) and relapse of pulmonary thromboembolism when joined with treatment complications $24 \%$. Half of deaths occurred in the first year of follow up, showing a shortened survival those patients with cancer $(p=0.02)$ and patients with relapses of pulmonary embolism ( $p=0.06)$. Beyond the first year, mortality declines to a rate of $10 \%$ per year mainly because of cardiovascular causes.

Mortality associated factors were age $>75$ years $(p<0.001)$ gender female $(p<0.01)$, a delayed admission and treatment from the beginning of symptoms $(p<0.05)$, higher $L D H$ level $(p<0.01)$ and coexistence of cancer $(p<0.05)$. In logistic-regression analysis age, delayed admission and treatment and higher LDH levels were predictors of long-term death.

Conclusions: Patients with pulmonary embolism show a high mortality rate, with a critical period during the first year after index episode, being deaths associated to cancer and to a composite of relapse of venous thromboembolic disease and bleeding complications. Mortality rate beyond the first year declines, being deaths explained because of cardiovascular causes.

An advanced age, a delayed diagnosis and treatment and serum LDH may predict long-term mortality.

KEY WORDS: Pulmonary thromboembolism.Venous thromboembolic disease. Long-term clinical course. Clinical epidemiology. 
Echegaría Agara M, Alonso Martínez JL, Urbieta Echezarreta MA, Abínzano Guillén ML, González Arencibia C, Solano Remírez, M. Tromboembolismo pulmonar: evolución a largo plazo y epidemiología clínica. An Med Interna (Madrid) 2003; 20: 451-456.

\section{INTRODUCCIÓN}

La enfermedad tromboembólica venosa, y su manifestación más grave el tromboembolismo pulmonar, es uno de los procesos patológicos vasculares más frecuentes, con una tasa importante de casos no diagnosticados (1). Hay mucha información en la literatura médica actual sobre los métodos diagnósticos, guías clínicas para el diagnóstico y tratamiento (2-4) y se está desarrollando un importante campo de trabajo en la investigación de los factores que conducen a hipercoagulación y trombofilia $(5,6)$.

Otros temas de interés en el campo de la enfermedad tromboembólica venosa radican en la evolución a largo plazo de este proceso, concretamente el diagnóstico de nuevas neoplasias, la tasa de accidentes hemorrágicos durante la profilaxis secundaria y la recidiva de la enfermedad. No obstante estos aspectos de la enfermedad han recibido menor atención en la literatura, conociéndose que la incidencia de cáncer tras el diagnóstico de enfermedad tromboembólica está incrementada entre 4-39\% (7), por término medio un 10\% (8) en aquellos pacientes sin causa conocida para la enfermedad, que la tasa de hemorragia grave es también aproximadamente del $10 \%$ (9) y la tasa de recidiva variable entre 3 y $28 \%$ (10-12).

El objetivo de nuestro estudio es conocer la evolución de los pacientes tras un ingreso índice por tromboembolismo pulmonar, la tasa de diagnostico de cáncer, la de recidiva de la enfermedad tromboembólica, y la de accidentes hemorrágicos e intentar descubrir factores de riesgo de mortalidad.

\section{MATERIAL Y MÉTODOS}

Se inició en mayo de 1992 un estudio de seguimiento de pacientes diagnosticados de tromboembolismo pulmonar agudo en una única área clínica de Medicina Interna de 8 camas que se ha llevado a cabo hasta diciembre-2002, con el objetivo de observar la supervivencia de los pacientes, las recidivas de la enfermedad tromboembólica, la tasa de hemorragias mayores y la aparición de nuevas neoplasias.

El seguimiento se realizó a través de contactos telefónicos con el paciente, o con los familiares del mismo en caso de haber fallecido, y a través del sistema informático de registro de pacientes y episodios clínicos del Servicio Navarro de Salud identificando los ingresos sucesivos, los diagnósticos sucesivos y la causa de la muerte.

Se define la hemorragia mayor como aquel episodio hemorrágico en el paciente anticoagulado que precisa atención en el servicio de urgencias o transfusión. Interpretamos como recidiva del tromboembolismo pulmonar aquellos casos bien definidos de recidiva del tromboembolismo y también aquellos casos de muerte súbita acompañados de disnea.

Nueva neoplasia se define como la que se diagnostica en el seguimiento del paciente y no se había diagnosticado previamente. Además constatamos la prevalencia total de cáncer incluyendo aquellos pacientes ya diagnosticados.
A partir de 1997 se midió nivel plasmático de dímero D por ELISA.

Análisis estadístico. Las variables continuas se expresan como media y como coeficiente de dispersión se utiliza la desviación estándar. Se utiliza la t de Student para comparación de medias con distribución normal y la U de Mann Whitney para aquellas con distribución no paramétrica. Las variables categóricas se expresan en porcentajes y se utiliza la comparación de proporciones y la prueba de ji al cuadrado para comparación de estas variables.

Calculamos las curvas de supervivencia con el método de Kaplan-Meier, y comparamos las curvas de supervivencia con la prueba de log-rank.

Para la predicción de supervivencia/mortalidad utilizamos un modelo de regresión logística paso a paso añadiendo variables que en general hubieran demostrado significación en los métodos bivariantes con una probabilidad mínima del 20\% ajustando las variables participantes hasta conseguir una probabilidad $<0,05$.

Se considera significación estadística un valor de $\mathrm{p}<0,05 \mathrm{y}$ tendencia estadística un valor de $\mathrm{p}<0,1$.

\section{RESULTADOS}

Se registraron 116 pacientes diagnosticados de tromboembolismo pulmonar con una edad media de $72 \pm 11$ años, varones $57(54 \%)$ y mujeres $49(46 \%)$. Cuatro $(3,7 \%)$ pacientes fallecieron durante el episodio primero de hospitalización.

Como profilaxis secundaria habían seguido tratamiento con heparina de bajo peso molecular 22, con acenocumarina 78 y por estar contraindicados los anticoagulantes, con antiagregación 4 pacientes (en dos se colocó filtro de cava). Noventa y cinco (94\%) de los pacientes cumplieron un mínimo de 6 meses de anticoagulación.

Los pacientes fueron seguidos durante un tiempo medio de $31,81 \pm 31,23$ meses (rango de 1 a 127), con $73(71,5 \%$ ) seguidos durante más de 12 meses y 43 (37\%) seguidos durante más de 24 meses. Diez $(8,6 \%)$ pacientes fueron perdidos durante el seguimiento, quedando la muestra por tanto en 102 pacientes.

Hubo $38(37,25 \%)$ fallecimientos en el seguimiento que ocurrieron 21,86 $\pm 24,76$ meses tras el diagnóstico. Fallecieron $14(37 \%)$ hombres y $24(63 \%)$ mujeres $(\mathrm{p}<0,01)$. Las causas del fallecimiento se reflejan en la tabla I. En total 9 pacientes $(24 \%)$ fallecieron como consecuencia de recidiva del tromboembolismo o como consecuencia de tratamiento (hemorragia cerebral). Diecinueve $(50 \%)$ de los pacientes fallecieron durante el primer año de seguimiento, y posteriormente un $10 \%$ por año. Las causas de fallecimiento durante el primer año se aprecian en la tabla I, suponiendo el 58\% la recidiva, la hemorragia o el cáncer.

Presentaron hemorragia mayor $11(10,4 \%)$ pacientes: cerebral 3 , digestiva 4 , retroperitoneal 1 , muscular 2 , epistaxis masiva 1 paciente. 
TABLA I

\begin{tabular}{lcc}
\multicolumn{4}{c}{ TABLA I } \\
$\begin{array}{lcc}\text { CAUSAS DE M UERTE EN PACIENTES CON } \\
\text { TRO M BO EM BO LISM O PULM O NAR }\end{array}$ \\
\hline Proceso & $\mathrm{n.} .(\%)$ & M uerte a los 12 meses \\
\hline Impreciso & $8(21)$ & $3(8 \%)$ \\
ACVA no hemorrágico & $5(13)$ & $3(8 \%)$ \\
Hemorragia cerebral & $3(8)$ & $2(5 \%)$ \\
M uerte súbita con disnea & $3(8)$ & $2(5 \%)$ \\
TEP recidivante & $3(8)$ & $2(5 \%)$ \\
Cáncer & $12(32)$ & $5(13 \%)$ \\
Insuficiencia cardiaca & $2(5)$ & $1(2,6 \%)$ \\
Insuficiencia renal & $2(5)$ & $1(2,6 \%)$ \\
\hline
\end{tabular}

Cáncer: Pulmón, Uno cada uno: ovario, linfoma, hígado, vejiga, próstata, Páncreas, colon, mama.

TEP: tromboembolismo pulmonar.

Sufrieron recidiva de la enfermedad tromboembólica 17 $(16,75 \%)$ pacientes: como tromboembolismo 12 y como trombosis venosa profunda 5 pacientes. Tres pacientes adicionales presentaron una muerte súbita con disnea en su domicilio lo que ofrece una tasa de recidiva total del 19\%. El tiempo de recidiva de la enfermedad tromboembólica fue de 22,64 \pm 24,57 meses (rango 1-73) (Fig. 1).

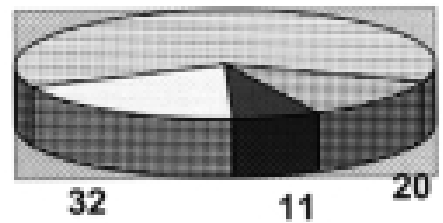

\begin{tabular}{|l|}
\hline Recidiva \\
$\square$ Hemorragia \\
$\square$ Cáncer \\
$\square$ Total TEP
\end{tabular}

Fig. 1. Distribución de eventos en pacientes con tromboembolismo pulmonar.

Se diagnosticaron $14(13,7 \%)$ nuevas neoplasias en el seguimiento: carcinomas pulmonares 4 , próstata 2 , vejiga 2 , colon 1, páncreas 1, ovario 1, mama 1, hígado 1, renal 1. En total la tasa de cáncer asociado al tromboembolismo pulmonar fue de 32 pacientes (31\%) con 18 pacientes ya diagnosticados previamente de neoplasia. En la figura 2 se puede apreciar la distribución total de neoplasias y las de nuevo diagnóstico.

\section{ANÁLISIS DE SUPERVIVENCIA}

En la tabla II se aprecian los factores que fueron diferentes en los pacientes que sobrevivieron respecto de los fallecidos.

La tasa de mortalidad por tromboembolismo fue mayor en las mujeres que en los hombres, y no parece debido al factor edad: edad de los hombres fallecidos 77,85 \pm 9,20 años, edad de las mujeres fallecidas 77,04 \pm 5,93 años (p no significativa).

Las curvas de supervivencia no mostraron diferencias al comparar los pacientes con y sin cáncer asociado, con accidentes hemorrágicos y con recidiva de la enfermedad tromboembólica (Fig. 3) a lo largo de todo el seguimiento. Sin

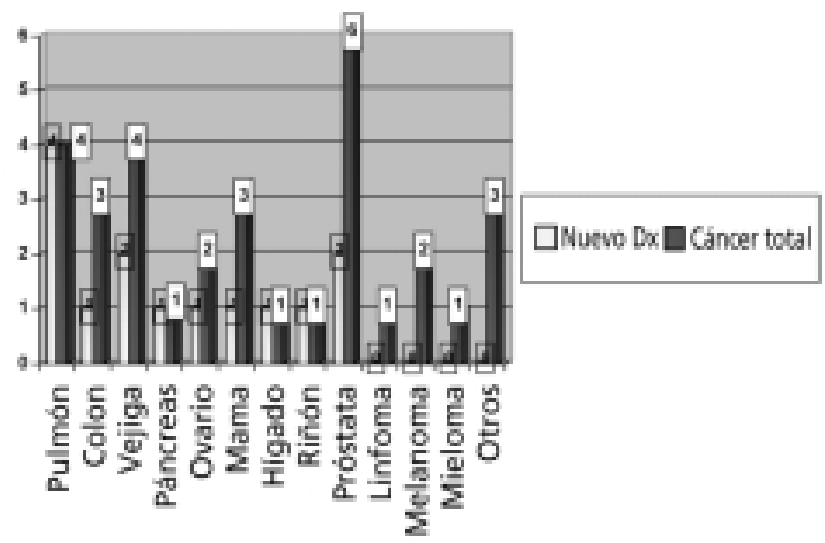

Fig. 2. Distribución de cáncer total y nuevas neoplasias en pacientes con tromboembolismo pulmonar.

\section{TABLA II}

DIFERENCIAS ENTRE SUPERVIVENCIA Y M UERTE DE LOS PACIENTES CON TRO M BO EM BO LISM OS PULM O NARES

\begin{tabular}{llll}
\hline & Supervivencia & muerte & $\mathrm{p}$ \\
\hline Edad & $68,95 \pm 12,5$ & $77,34 \pm 7,20$ & $<.001$ \\
Sexo (\% V/M) & $72 / 47$ & $25 / 49$ & $<.01$ \\
Frec. de pulso & $86,73 \pm 15,42$ & $92,94 \pm 13,67$ & $<.05$ \\
TAS (mm Hg) & $123,9 \pm 18,28$ & $118,15 \pm 19,81$ & .06 \\
Frec. respiratoria & $25,03 \pm 6,25$ & $28,23 \pm 5,18$ & $<.01$ \\
Cáncer total (\%) & 20 & 45 & $<0.5$ \\
Hemorragia (\%) & 6 & 18 & .08 \\
Recidiva ETE (\%) & 9 & 21 & NS \\
Días de clínica & $8,17 \pm 12,19$ & $13,69 \pm 12,99$ & $<.05$ \\
LDH (U/1) & $231 \pm 84$ & $292 \pm 100$ & $<.01$ \\
\hline
\end{tabular}

ETE: enfermedad tromboembólica

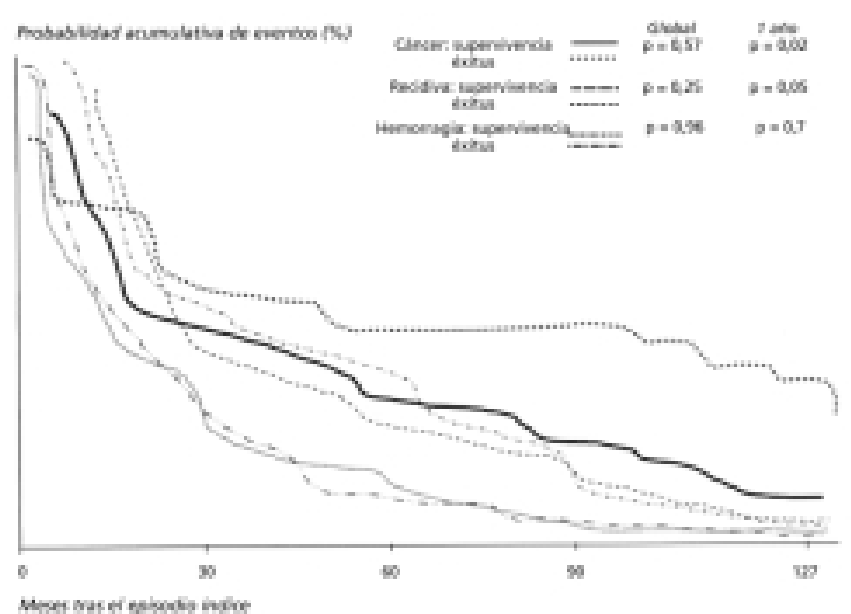

Fig. 3. Curvas de supervivencia en pacientes diagnosticados de tromboembolismo pulmonar para cáncer asociado, recidiva y hemorragia. 
embargo al analizar el primer año de seguimiento la supervivencia en los pacientes con cáncer es menor que en los pacientes sin cáncer $(\mathrm{p}=0,02)$, y se apreciaba también una tendencia a menor supervivencia en los pacientes con recidiva de la enfermedad tromboembólica $(\mathrm{p}=0,06)$. No tenían efecto en la supervivencia las hemorragias.

Aunque en el grupo total la proporción de hemorragias sólo tendía a ser mayor en el grupo de los fallecidos, al considerar solo estos últimos la proporción de sangrado fue mayor $(64 \%)$ que los que no presentaron hemorragia $(33 \%)(\mathrm{p}<0.05)$.

No se encontraron predictivos de recidiva ni de hemorragia mayor entre la edad, sexo, datos gasométricos, frecuencia respiratoria, cardiaca, LDH, tratamiento con cumarina o heparina de bajo peso molecular, tiempo de seguimiento ni diagnóstico de cáncer asociado.

El nivel de dímero D se midió en 63 pacientes y su nivel en el episodio índice fue menor en los pacientes con hemorragia $(1,47 \pm 1,07)$ que en los que no tuvieron complicaciones hemorrágicas $(5,55 \pm 6,34)(\mathrm{p}<0,01)$. Los pacientes con un nuevo diagnóstico de cáncer tuvieron un nivel de dímero $\mathrm{D}$ de $7,06 \pm 4,25$, mientras que los pacientes sin cáncer asociado fue de 4,94 $\pm 6,84(\mathrm{p}=0,03)$. El nivel de dímero $\mathrm{D}$ en el episodio índice fue similar en los pacientes que recidivaron y los que no.

En el modelo de regresión logística las variables que predijeron mortalidad fueron la edad, los días desde el inicio de la clínica hasta el ingreso en el episodio índice y el nivel de LDH en suero medida en el episodio índice (Tabla III).

\section{DISCUSIÓN}

La literatura médica disponible sobre la enfermedad tromboembólica venosa es muy amplia, en particular en lo referente a diagnóstico, tratamiento y factores de riesgo (1-5). Sin embargo los datos disponibles sobre la evolución a largo plazo son menos abundantes en este campo, sobre todo los datos que hacen referencia a la tendencia a la recidiva, las complicaciones del tratamiento y la incidencia de diagnostico de nuevas neoplasias.

Aunque existen guías de consenso para la profilaxis secundaria de la enfermedad tromboembólica venosa, el tiempo de anticoagulación en la forma idiopática, aunque se recomienda que sea de 6-12 meses, no está bien establecido (11). En general una duración de la anticoagulación superior a tres meses, se asocia a una disminución de las recidivas de la enfermedad a costa de incrementar el riesgo de hemorragia, cesando el beneficio en cuanto cesa la anticoagulación (13). Se ha señalado que un aumento progresivo del dímero $\mathrm{D}$ durante la anticoagulación, y cuando se utiliza heparina no fraccionada, un retraso en alcanzar el objetivo de doblar el

\section{TABLA III}

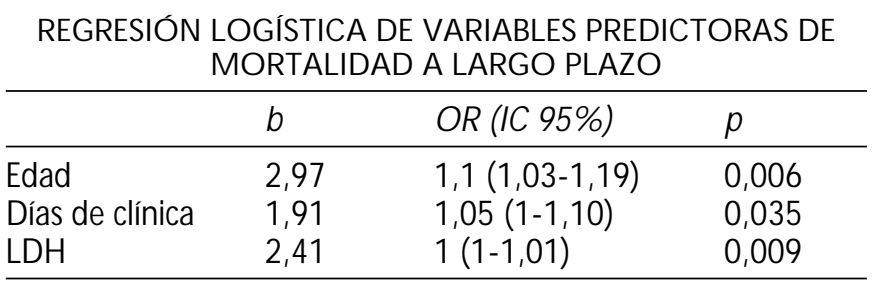

tiempo basal de aPTT, se asocia a mayor riesgo de recidiva de la enfermedad $(10,14)$. La incidencia de recidivas de la enfermedad es variable entre trabajos cifrándose entre 2,7 y $28 \%$ en dependencia de los factores de riesgo y de los antecedentes previos de enfermedad tromboembólica. En general los pacientes con cáncer, afectación venosa proximal por trombosis y el haber tenido previamente un episodio de enfermedad tromboembólica se acompañan de mayor tasa de recurrencia de la enfermedad $(10,12,15)$, mientras el sexo femenino, la coexistencia de cáncer y la edad avanzada se asocian a mayor tasa de hemorragia $(9,15)$. Además en estos pacientes la tasa de recurrencia fatal puede ser de hasta el $28 \%$, siendo mucho más frecuente en los pacientes en quienes la enfermedad tromboembólica venosa se manifestó como tromboembolismo pulmonar (16). Se considera que el riesgo de recurrencia persiste durante muchos años (17) tras el episodio índice, aunque otros autores opinan que el riesgo disminuye sensiblemente con el paso del tiempo $(16,18)$.

Por otra parte la incidencia de cáncer de nuevo diagnóstico también parece estar incrementada en los pacientes con enfermedad tromboembólica de causa idiopática mostrando cifras variables entre 3,9 y $36 \%$ según el tiempo de seguimiento (17\% al año, 39\% a los 5 años) (10-12). Los mecanismos por lo que una neoplasia puede dar lugar a enfermedad tromboembólica son variados: las células tumorales pueden activar los sistemas de coagulación, las células mononucleares expresan y sintetizan sustancias procoagulantes en respuesta al tumor, algunos quimioterápicos pueden dañar las células endoteliales, el uso frecuente de accesos intravasculares es un factor precipitante bien identificado de enfermedad tromboembólica venosa en pacientes con cáncer (19).

El riesgo de cáncer no esta delimitado aciertos tipos de neoplasia, si no que virtualmente cualquier órgano y sistema puede verse afectado $(20,22)$. En series de nuestro país el carcinoma de próstata y el carcinoma colorrectal son las neoplasias más frecuentemente implicadas como diagnostico de neoplasia en la evolución de la enfermedad tromboembólica, mientras que el cáncer de mama, pulmón, útero y cerebro desarrollan la enfermedad tromboembólica como acontecimiento terminal $(23,24)$.

Aunque algunos autores concluyen que la anticoagulación oral puede tener un efecto antineoplasico (25), en la actualidad parece que existen pruebas acerca del efecto antitumoral de las heparinas de bajo peso molecular (disminución de la proliferación vascular, aumento de la supervivencia de pacientes tratados con heparinas de bajo peso molecular y menor incidencia de complicaciones hemorrágicas) $(26,27)$.

En nuestros datos, aunque la serie no es numerosamente muy amplia, el seguimiento se ha hecho durante un tiempo medio de 2,5 años, tiempo que nos parece suficientemente prolongado para observar los acontecimientos planteados como objetivos (Prevalencia de cáncer y nuevas neoplasias, recidiva de la enfermedad, accidentes hemorrágicos y mortalidad total) lo que puede servir para hacernos idea del problema en nuestro medio de trabajo.

Es de señalar que la enfermedad tromboembólica venosa no es una enfermedad de pacientes jóvenes, siendo la edad un factor de riesgo probablemente de enfermedad y como discutiremos luego de mortalidad.

La principal causa de muerte en nuestra serie es el cáncer, bien como enfermedad ya diagnosticada, siendo el tromboembolismo un evento acompañante, o bien como de nuevo 
diagnóstico. El espectro de neoplasias es variado, siendo los más frecuentes el carcinoma pulmonar y el carcinoma de próstata. La limitación numérica de los pacientes puede sesgar el espectro de la neoplasia de nuevo diagnostico, pero estos tipos de cáncer son dos neoplasias de las de mayor incidencia y prevalencia en la población general, lo que puede indicar que no existe un tipo determinado de neoplasia asociada al tromboembolismo sino que cualquier cáncer puede asociarse con enfermedad tromboembólica.

En nuestros datos, la segunda causa de mortalidad es el propio tromboembolismo. Cuando consideramos el tromboembolismo clínicamente definido y la muerte súbita con disnea como causa de muerte junto a complicaciones hemorrágicas del tratamiento, explican la cuarta parte de los fallecimientos, lo que supone que el tratamiento de la enfermedad no es óptimo, mostrando una mortalidad elevada, por lo que se deben buscar alternativas terapéuticas más eficaces y con fármacos más seguros, sin un rango terapéutico tan estrecho.

La disminución de la supervivencia en pacientes con cáncer y con recidiva de la enfermedad tromboembólica en nuestros pacientes, ocurre fundamentalmente durante el primer año del episodio índice, igualándose las curvas de supervivencia en plazos de tiempo más largos. Este tipo de curva de supervivencia, con una fuerte pendiente de declinación inicial para estabilizarse posteriormente es similar a la publicada para otros países (18). Por lo tanto, la principal mortalidad ocurre durante el primer año de seguimiento disminuyendo posteriormente y manteniéndose estable durante los años siguientes a una frecuencia aproximada de $10 \%$ por año. Las causas de mortalidad inicial estarían relacionadas con la recidiva de el enfermedad tromboembólica venosa y con el cáncer, siendo la mortalidad posterior no relacionada con la enfermedad tromboembólica o desencadenante. En conjunto la mitad de los pacientes fallecen como consecuencia del tromboembolismo, del tratamiento o de cáncer y lo hacen durante el primer año.

En el modelo de regresión logística planteado las variables que mejor explicaban la mortalidad eran la edad, los días de clínica previos al ingreso, diagnóstico e inicio del tratamiento y un nivel de LDH más elevado en el episodio índice. Nos parece de gran importancia el hecho de que en algunos niveles asistenciales el tromboembolismo pulmonar no se considere como posibilidad diagnóstica de pacientes con diferentes combinaciones de disnea, dolor toracico y síncope, siendo diagnosticados en general de infección respiratoria o insuficiencia cardíaca motivando un retraso en el diagnóstico y tratamiento que según nuestros datos pueden tener implicaciones pronósticas.

No hemos encontrado indicadores clínicos de recidiva o sangrado a pesar de que en la literatura están delimitados la edad, el sexo femenino y la existencia de cáncer como factores de riesgo de hemorragia. El único dato que mostró diferencias fue el nivel de dímero $\mathrm{D}$ en el episodio índice antes de comenzar la anticoagulación que fue más bajo en los pacientes que posteriormente sangraron. Este dato es de difícil interpretación, y necesitaría ser confirmado en estudios epidemiológicos más amplios antes de concederle relevancia clínica.

Aunque la tasa de recidiva fue del $20 \%$ ocurriendo 21 meses después del episodio índice, de nuevo tampoco encontramos factores clínicos asociados a las recidivas, aunque el relativo tamaño pequeño de la muestra, y el no incluir en nuestros pacientes estudio de trombofilia puede mediatizar los resultados e impedir obtener conclusiones válidas en este campo.

El tanto más importante del presente trabajo, nos parece que supone determinar que los pacientes seguidos tras un episodio de tromboembolismo pulmonar tienen una mortalidad elevada, sobre todo por cáncer y por el tromboembolismo o su tratamiento, pasando un periodo crítico de un año tras el episodio de tromboembolismo, a partir del cual va disminuyendo la mortalidad posterior a cifras estables por año, y siendo la mortalidad tardía debida a causas no relacionadas con el episodio índice. Fallecen principalmente mujeres, de edad avanzada, con retraso en el diagnóstico y tratamiento del episodio índice y unos niveles de LDH elevados.

\section{Bibliografía}

1. Dalen JE. Pulmonary embolism; What have we learned since Virchow? Natural history, pathophysiology, and diagnosis. Chest 2002; 122: 1440-56

2. Musset D, Parent F, meyer G, maitre S, Girard P, leroyer C, et al. Diagnostic strategy for patients with suspected pulmonary embolism: a prospective multicentric outcome study. Lancet 2002; 360: 1914-20.

3. Kruip MJHA, Slob MJ, Schijen JHEM, van der Heul C Büller HR. Use of a clinical decision rule in combination with D-Dimer concentration in diagnostic work up of patients with suspected pulmonary embolism. Arch Intern Med 2002: 162: 1631-5.

4. Chagnon I, Bounameaux H, Aujesky D, Roy PM, Gourdier AL, Cornuz $\mathrm{J}$, et al. Comparison of two clinical prediction rules and implicit assessment among patients with suspected pulmonary embolism. Am J Med 2002; 113: 269-75.

5. Kelly J, Rudd A, Lewis RR, Hunt BJ. Plasma D dimers in the diagnosis of venous thromboembolism. Arch Intern Med 2002;162: 747-54.

6. Horne III MDK. The dark side of deep venous thrombosis: the failure of anticoagulation. Am J Med 2001; 110: 589-90.

7. Prins MH, Hettiarachchi RJ, Lensing AW, Hirsh J. Newly diagnosed malignancy in patients with venous thromboembolism. Search or wait and see?. Thromb Haemost 1997;78: 121-5.

8. Otten HM, Prins MH. Venous thromboembolism and occult malignancy. Throm Res 2001; 102: 187-94.

9. Landefeld CS, Beyth R. Anticoagulant-related bleeding: clinical epidemiology, prediction and prevention. Am J Med 1993;95:315-328.

10. Hutten BA, Prins MH, Gent M, Ginsberg J, Tijssen JG, Büller HR. Incidence of recurrent thromboembolism and bleeding complications among patients with venous thromboembolism in relation to both malignancy and achieved international normalized ratio: a retrospective analysis. Clin Oncol 2000; 18: 3078-83.

11. Prandoni P. Long-term clinical course of proximal deep venous thrombosis and detection of reccurent thrombosis. Semin Thromb Haemost 2001; 27: 9-13.

12. Hansson PO, S:orbo J, Ericksson H. Recurrent venous thromboembolism after deep venous thrombosis. Arch Intern Med 2000; 160: 769-74.

13. Agnelli G, Prandoni P, Santamaría MG, Bagatella P, Iorio A, Bazzan M, et al. Three months versus one year of oral anticoagulation for idiopathic deep venous thrombosis. Warfarin optimal duration italian trial investigators. N Engl J Med 2001; 345: 165-9.

14. Palareti G, Legnani C, Cosmi B, Guazzaloca G, Pancani C, Cocheri S. Risk of venous thromboembolism recurrence: high negative prediction 
value of D-Dimer performed after oral anticvoagulation is stopped. Throm Haemost 2002; 87: 7-12.

15. Kruijer PM, Hutten BA, Prins MH, Büller HR. prediction of the risk of bleeding during anticoagulation for venous thromboembolism. Arch Intern Med 1999; 159: 457-60.

16. Douketis JD, Keaton C, Bates S, Duku EK, Ginsberg JS. Risk of fatal pulmonary embolism in patients with treated venous thromboembolism. JAMA 1998; 279: 458-62.

17. Prandoni P, Lensing AWA, Cogo A, Cuppini S, Villalta S, Carta M, et al. The long-term cliical course of acute deep venous thrombosis. Ann Intern Med 1996; 125: 1-7

18. Beyth RJ, Coher AM, Landefeld CS. Long-term outcomes of deep-vein thrombosis. Arch Intern Med 1995; 155: 1031-7.

19. Prandoni P, Pccioli A, Girolami A. Cancer and venous thromboembolism: an overview. Haematologica 1999; 84: 437-45.

20. Piccioli A, Prandoni P. Venous thrombosis as first manifestation of cancer. Acta Haematol 2001; 106: 13-7.
21. Rickles FR, Lovine MN. Venous thromboembolism in malignancy and malignancy in occult thromboembolism. Haemostasis 1998; (Supl) 3: 43-9.

22. Prandoni P. Cancer and thromboembolic disease: How important is the risk of thrombosis. Cancer Trat Rev 2002; 28: 133-6.

23. Monreal M, Prandoni P. Venous thrombosis as first manifestation of cancer. Semin Thromb Hemost 1999; 2s: 131-6.

24. Monreal M, Fernández-Llamazares J, Perandreu J, Urrutia A, Sahuquillo JC, Contel E. Occult cancer in patients with venous thromboembolism: which patients, which cancers. Thromb Haemost 1997; 78: 1316-8.

25. Shulman S, Lindmarker P. Incidence of cancer after prophylaxis with warfarin against recurrent venous thromboembolism. Duration of anticoagulant trial. N engl J Med 2000; 342: 1953-8.

26. Ornstein D, Zacharsky L. The use of heparin for treating human malignancies. Haemostasis 1999; 29 (Supl. s1): 48-60.

27. Engelberg H. Actions of heparin that may affect the malignancy process. Cancer 1999; 85: 257-72. 